\title{
Globalbudget ante portas - die Schweiz blockiert sich selbst
}

Marcus M. Maassen

Prof. Dr. med., Mitglied FMH

Seit dem 1.1. 2018 gilt die vom Bundesrat mit subsidiärer Kompetenz eingesetzte, zweite national einheitliche Tarifstruktur TARMED. Unter den Ärzten wird dieser neue Tarif, der in den Medien [1] massiv kritisiert worden ist, offen als "Berset-Tarif» bezeichnet. Viele Fachbereiche, von der Allgemeinmedizin über die Dermatologie bis hin zur Psychiatrie sind massiv von den einschneidenden, teilweise existenzgefährdenden Massnahmen betroffen. Wenn in einigen Fachbereichen, wie etwa in der Dermatologie, Vergütungen ärztlicher Leistungen um bis zu 30\% oder in der Konsiliarpsychiatrie um ca. $15 \%$ gekürzt werden, wird dies nicht ohne Auswirkungen auf die Arzt-Patient-Zeit und die von den Ärzten angestellten Medizinalpersonen sein. Der Berset-Tarif wirkt sich wirtschaftlich so nachteilig auf weite Teile der ärztlichen Tätigkeit aus, dass er das Gebot der Sachgerechtigkeit verletzt und somit rechtswidrig ist.

Das Bundesamt für Gesundheit (BAG) rechtfertigt seine Eingriffe zur angeblichen Kostendämpfung damit, dass «eine Mengenausweitung» zu korrigieren sei. Interessant dabei ist, die technokratische Bezeichnung einer «Menge» bei der Versorgung der Patienten zu hinterfragen. Wenn ein Arzt statt früher 4 Patienten pro Stunde neu 6 Patienten pro Stunde behandelt, dann sind dies Mehrbehandlungen von kranken Men-

\section{Résumé}

La seconde structure tarifaire homogène au niveau national TARMED, ouvertement appelée "tarif Berset», est entrée en vigueur en janvier. $\mathrm{Ce}$ tarif est inapproprié et a déjà entraîné une pénurie régionale de soins médicaux. Une raréfaction supplémentaire des prestations médicales se dessine en outre avec l'initiative sur la santé du PDC, dont l'objectif est d'encourager l'instauration d'un budget global. II a pu être démontré dans d'autres pays que ce type de budget ne réduit pas les coûts et n'améliore pas la qualité. Nous, les médecins, devons unir nos forces contre un budget global! Si les partenaires tarifaires ne trouvent pas d'accord, I'OFSP fera usage de ses compétences subsidiaires. Nous devrions suivre la proposition de notre président (BMS 2018;99;0102) de nous focaliser sur une solution et de présenter un tarif commun, même s'il implique des sacrifices. Nous avons une responsabilité à l'égard de notre liberté. schen, die medizinische Hilfe benötigen. Natürlich wird mehr Zeit und Arbeit für diese Patienten benötigt. Es handelt sich aber um Menschen und nicht um eine Menge. Wenn die medizinische Mehrarbeit als Menge bezeichnet wird, dann haben wir zwar eine technokratisch-dirigistische Bezeichnung, die jedoch beschämend ist angesichts der verantwortungsvollen ärztlichen Tätigkeit. Wir Ärzte wollen nicht - wie von manchem Sparfuchs behauptet -Gesundheit als Ware verkaufen, sondern wir wollen die Gesundheit unserer Patienten wiederherstellen. Wir wollen heilen und helfen. Dafür haben wir studiert und eine lange Ausbildung mit vielen Entbehrungen über uns ergehen lassen.

\section{Trotz Globalbudget keine Kostendämp- fung und keine Qualitätsverbesserung}

Die vom Bundesrat eingesetzte Expertenkommission erarbeitet Massnahmen auf der Grundlage der Auswertung internationaler Erfahrungen (D, NL). Diese internationalen Erfahrungen sind nicht der Weisheit letzter Schluss. Fakt ist, dass die OECD einen internationalen Ländervergleich (compareyourcountry.org) publiziert hat, der aufzeigt, dass in den Niederlanden trotz Globalbudget keine Kostenvorteile realisiert wurden.

Wenn schon die Kosten durch ein Globalbudget nicht gesenkt werden, stellt sich die Frage, ob die Qualität der Gesundheitssysteme in den Niederlanden oder in Deutschland besser ist. Auch hier kann man auf solide Studien zurückgreifen. Im internationalen Vergleich von 195 Ländern über einen Zeitraum von 1990 bis 2015 belegt die Schweiz den dritten Platz, deutlich vor den Niederlanden (Platz 9) und Deutschland (Platz 20) [2]. Fazit: Trotz Globalbudget keine Qualitätsverbesserungen.

Während die Ärzte für jeden Eingriff rechtlich verpflichtet sind, über Risiken und Nebenwirkungen aufzuklären, agiert die «Expertengruppe Kostendämpfung» autonom, ohne auf Risiken und Nebenwirkungen hinzuweisen. Wohin haben denn die staatsmedizinischen Eingriffe in Deutschland geführt? Wie verhält 
es sich denn mit Wartezeiten auf einen Facharzttermin in Deutschland? Wieviel Zeit steht einem Arzt in einer Praxis für die Behandlung eines Patienten zur Verfügung? Mit keinem Wort wird der Schweizer Bürger über die Risiken der Vorschläge der Expertenkommission zur Kostendämpfung des BAGs informiert. Fakt ist: Im «grossen Kanton» Deutschland wird der Zustand

Heute hat kein Allgemeinarzt in Deutschland Zeit, sich 15 Minuten mit einem obligatorisch versicherten Patienten zu beschäftigten.

des Gesundheitssystems von den politisch Verantwortlichen und den sogenannten Gesundheitsexperten beschönigt. Seit 30 Jahren gibt es in Deutschland ein Globalbudget. Die Qualität der Versorgung hat sich seit dieser Zeit nicht verbessert, sondern verschlechtert. Heute hat kein Allgemeinarzt in Deutschland Zeit, sich mehr als 15 Minuten im Durchschnitt pro Sprechstundentag mit einem obligatorisch versicherten Patienten zu beschäftigten. Die 5-Minuten-Medizin ist zur Regelzeit geworden. Termine werden pro Patient möglichst nur einmal pro Quartal vergeben. Wartezeiten auf Arzttermine von drei bis sechs Monaten auf einen Facharzttermin gehören zum Normalzustand. Gleichzeitig fordert die Politik mit einer Selbstverständlichkeit von den Ärzten, dass sich die Qualität der Leistungen trotz der massiven Kürzungen nicht verschlechtert. Die Politik leitet Massnahmen ein, die unweigerlich zu einem Rationieren führen werden, delegiert dies aber an den Arzt. Glaubwürdigkeit sieht anders aus.

Ganz zu schweigen von der Zukunft der Versorgungssicherheit und der Motivation für die jungen Kollegen. Der induzierte Nachwuchsmangel bei konservativ tätigen Augenärzten verursacht in Deutschland Wartezeiten auf einen Termin jenseits der Halbjahresfrist [3]. Warum enthält die «Expertenkommission Kostendämpfung» dem Schweizer Bürger derartige Risiken ihrer Vorschläge vor?

\section{Globalbudget führt zu Wartezeiten auf Termine}

Wird sich diese Entwicklung auch in der Schweiz fortsetzen? Die Gutgläubigen und Träumer rechnen nicht mit solchen Entwicklungen. Die Realität sieht leider anders aus. Der massive subsidiäre Eingriff des Bundesrates in die Tarife hat beispielsweise bei den Handchirurgen zu betriebswirtschaftlich nicht rechtfertigbaren Einnahmenverlusten geführt, sodass sich in Genf die freiberuflich tätigen Handchirurgen gefolgt von Gynäkologen, Geburtshelfern und Orthopäden weigern, ambulante Eingriffe durchzuführen. Diese Ein- griffe werden dann in einer öffentlichen Klinik durchgeführt werden müssen. Wer allerdings glaubt, dass die Kapazitäten der Spitäler für ambulante Eingriffe unbegrenzt sind, der irrt. Wenn aber die Kapazität nicht erbracht werden kann, werden für die Patienten Wartezeiten auf Behandlungstermine resultieren, genauso wie in Deutschland. Tarifkürzungen für ambulante Eingriffe haben in Deutschland genau den Effekt gehabt, dass viele Ärzte ambulant durchführbare Resektionen nicht mehr anbieten können, weil die Vergütungen nicht die Fixkosten decken. In Süddeutschland beträgt beispielsweise die Wartezeit für Termine an Zentren für die Nachresektion histologisch gesicherter maligner Melanome oder Plattenepithelkarcinome der Haut über 6 Wochen. Für den betroffenen Patienten ist dies eine sehr lange Zeit, da er weiss, dass der Tumor in dieser Zeit potentiell metastasieren kann. Auch in der Schweiz können unter dem aktuellen Berset-Tarif in vielen Bereichen Leistungen nicht kostendeckend angeboten werden. Wenn das Beispiel der Handchirurgen Schule macht, werden ganze Sektoren der ambulanten Medizin mit Wartezeiten auf Eingriffe konfrontiert werden. Wirkt nicht die Kürzung der ambulanten OP- Leistungen gerade dem vom BAG proklamierten Ziel einer Leistungsverlagerung stationär zu ambulant diametral entgegen?

\section{Globalbudget führt zu temporär geschlos- senen Arztpraxen}

Sicher kalkuliert die «Expertengruppe Kostendämpfung» ein, dass wir Mediziner unsere Aufgaben gerne verrichten und auch mit einer niedrigeren Vergütung unsere Arbeit weiter leisten werden. Sicher ist es auch richtig, dass der Arztberuf ein hohes Mass an Ausbildung und ständiger Weiterbildung, persönlichem

\section{Das Kalkül der Gesundheitsökonomen könnte vielleicht aufgehen, dass die ärztliche Leistung auch günstiger einzukaufen sei.}

Engagement, Verzicht, Verpflichtung zur Rufbereitschaft nachts, an Wochenenden und an Feiertagen verlangt. Das Kalkül der Gesundheitsökonomen könnte vielleicht aufgehen, dass die ärztliche Leistung auch günstiger einzukaufen sei. Jedoch kann keiner von der Ärzteschaft angesichts der massiven Einschnitte in die Leistungsvergütung erwarten, dass wir für unsere Arbeit auch noch zahlen. Der Bundesrat beabsichtigt anscheinend, im Ausland bereits gescheiterte gesundheitsökonomische Massnahmen zu übernehmen, weil diese sich aufgrund angeblicher Expertenmeinungen bewährt haben. So wird auf die Schweizer Ärzte ein 
Zwang ausgeübt, ihrerseits ihr wirtschaftliches Überleben mit den gleichen Methoden zu gewährleisten, welche sich schon in Deutschland als einzig gängiger Ausweg erwiesen haben. In den letzten Wochen eines Quartals, wenn das definierte Budget erzielt worden ist, bleiben die Praxen geschlossen! Aus ökonomischen Gründen sind auch keine anderen Massnahmen möglich, denn die Personalkosten können ohne Einnahmen nicht bezahlt werden. Ist das Budget erschöpft, werden auch die letzten motivierten Ärzte ihre Patienten nicht mehr behandeln, denn die Praxen würden ansonsten defizitär arbeiten.

\section{Bevormundung der Patienten}

Wenn gleichzeitig die CVP mit ihrer gesundheitspolitischen Initiative fordert, dass die Gesundheitskosten nur 1,1-mal so stark steigen dürfen wie die Nominallöhne, bedeutet dies nichts anderes als die Einführung eines Globalbudget mit gesetzgeberischen Mitteln. In den Ländern, die ein Globalbudget haben, sind Rationierungen und verlängerte Wartezeiten auf Arzttermine nicht zu vermeiden. Dies ist eine Bevormundung der Patienten. Es kann so weit kommen, dass sich der Arzt bei multimorbiden Patienten pro Quartal nur einem Leiden widmen kann. Dies ist die Konsequenz, weil ja das Budget erschöpft ist. Diesen Sachverhalt

In den Ländern, die ein Globalbudget haben, sind Rationierungen und verlängerte Wartezeiten auf Arzttermine nicht zu vermeiden.

sucht man vergebens im Positionspapier der CVP. Begründet wird hingegen im Positionspapier der CVP die Notwendigkeit einer Kostenbremse mit den guten Erfahrungen, die man mit einer Schuldenbremse im Finanzbereich gemacht habe. Die spezifischen Besonderheiten des Gesundheitswesens (z.B. demographische Entwicklung, neue Therapieverfahren, innovative Medikamente) lassen sich jedoch nicht mit dem des Finanzbereiches vergleichen.

\section{Ein gutes Gesundheitssystem trägt dazu bei, «dass Menschen bei der Arbeit weni- ger fehlen und das längere Arbeitsleben besser bewältigen.»}

Eine Anfang Februar veröffentlichte Studie der RatingAgentur Moody's Investors Service demonstriert, dass bei den Bonitätsbewertungen europäischer Staaten ein besonderes Augenmerk auf die Gesundheitssektoren der jeweiligen Länder zu richten ist [4]. «In einem un- günstigen Szenario rechnet die EU-Kommission laut der Studie damit, dass die öffentlichen Ausgaben für das Gesundheitswesen von einem durchschnittlichen Anteil von 6,9\% am Bruttoinlandprodukt (BIP) der EULänder 2013 auf 9,5\% im Jahr 2060 steigen könnten. «Kürzungen im Gesundheitswesen alleine zögen folglich keine bessere Bonitätsbewertung nach sich, wenn sie sich in einem deutlichen Rückgang der Produktivität oder der sozialen Stabilität äusserten, schreibt Moody's. Schliesslich sollte ein gutes und zugängliches Gesundheitssystem dazu beitragen, dass Menschen bei der Arbeit weniger fehlen und das längere Arbeitsleben besser bewältigen (NZZ, 19.2.2018).»

Werden die Gesundheitskosten an die Nominallöhne resultiert ein Globalbudget mit einem verschlechterten Gesundheitssystem. Die CVP-Gesundheitsinitiative schadet somit dem Standort Schweiz. Die Ärzte sollten geeint ihre Patienten über die massiven Risiken und den Zwang, der mit der CVP-Gesundheitsinitiative verbunden ist, aufklären.

Jeder Franken, der durch die Politik des BAG und durch eine Realisierung der gesundheitspolitischen Initiative der CVP der Medizin vorenthalten werden würde, käme der Industrie und den grauen Eminenzen im Hintergrund zugute, weil das Geld ja bekanntlich nie verschwindet, sondern nur den Besitzer wechselt. Mit der Einführung eines Globalbudgets würde aber der Schalter auf "on" gestellt werden, der das Schweizer Gesundheitswesen in den Selbstzerstörungsmodus schaltet. Es entbehrt nicht einer gewissen Pikanterie, dass am 15.2.2018 auf der Frontseite der NZZ das «Luxusproblem der Schweiz», nämlich für das Jahr 2017 2,8 Milliarden Franken Überschuss in der ordentlichen Finanzierungsrechnung publiziert wurde, aber mit politischen Initiativen am höchsten Gut des Menschens, nämlich der Gesundheit, gespart werden soll.

\section{Zwang zur Übernahme der Verantwortung}

Sollte das BAG sich eine «verbindliche Zielvorgabe für das Kostenwachstum" auf die Fahne geschrieben haben, dann hat der Bürger ein Recht auf Klartext. Hinter den Vorstellungen des BAGs verbirgt sich nämlich ebenfalls nichts anderes als die schrittweise Einführung eines Globalbudgets. Und genau darum geht es: Herr Bundesrat Berset strebt durch die nicht von uns Ärzten zu beeinflussenden Tarifeingriffe an, dass die «Mengenausweitung» zunimmt, und er so weitere noch striktere Massnahmen ergreifen kann. So kommt das Globalbudget auf leisen Sohlen immer näher. Das perfide daran ist, dass der Berset-Tarif mit einer Verordnungsänderung formell umgesetzt wird. Daher be- 
steht für den Bürger keine Möglichkeit, Einfluss zu nehmen, z.B. in Form eines Referendums. Und natürlich klärt keiner der Gesundheitsökonomen darüber und über die Risiken eines solchen aktionistischen Systemeingriffs auf. Sehr bedauerlich für den Arzt, der einige Wochen vor Quartalsende ein Schild an der Praxistür anbringt, dass die Praxis bis zum Ende des Quartals geschlossen bleiben muss.

Hofft man darauf, dass von bürgerlicher Seite Aufklä-

\section{So kommt das Globalbudget auf leisen Sohlen immer näher.}

rung aus den Medien zu den potentiell versorgungsgefährdenden Massnahmen des BAG erfolgt, muss man leider mit einer gewissen Enttäuschung feststellen, dass auch in vielen Berichterstattungen ein Journalismus betrieben wird, der in weiten Teilen einer sachlich nicht zu rechtfertigenden Hexenjagd auf Ärzte gleichkommt.

\section{Die Schweiz blockiert sich selbst}

Die Vorschläge der «Expertengruppe Kostendämpfung» sind nicht geeignet, das Schweizerische Gesundheitssystem zu verbessern. Es wird zwar umgebaut, aber nicht verbessert. Der Administrationsaufwand im Gesundheitswesen wird massiv ansteigen. Medizinfremde Kosten, dysfunktionale, teure IT-Zwangsinvestitionen, eine Zunahme sog. Gesundheitsökonomen, die alle aus dem Budget finanziert werden müssen, werden zu einer Fehlallokation der Mittel und zu einem unschweizerischen Regulierungszwang führen. Für den prämienzahlenden Schweizer Bürger wird bei der Realisierung der Vorschläge der «Expertenkommission Kostendämpfung» des BAG aber weniger Zeit und Geld zur Verfügung stehen. Die Vorschläge der vom EDI eingesetzten, z.T. aus dem Ausland stammenden, Experten werden zu Veränderungen führen, die auf dem Rücken der Schweizer Patienten ausgetragen werden. Die Schweiz blockiert sich selbst.

\section{Einheit ist gefordert}

Wir Ärzte müssen geeint auftreten. Falls keine Einigung der Tarifpartner zustande kommen, wird das BAG seine subsidiären Kompetenzen durchsetzen. Wir sollten den Vorschlag unseres Präsidenten unterstützen [5], lösungsorientiert einen gemeinsamen Tarif vorzulegen, auch wenn dies mit Abstrichen verbunden ist. Wir haben eine Verantwortung für unsere Freiheit.



Literatur

1 Bersets Kunstfehler, Weltwoche, 2018, Nr. 08.18, 16-8.

2 The Lancet, Volume 390, Issue 10091, 15-21 Jul: 2017; 231-66

3 Schulte U. Globalbudget im Gesundheitswesen - ein Problem als Lösung. Schweiz Ärztezeitung. 2017;98(25):796-7.

4 Ohne Gesundheit ist alles nichts. NZZ,19.2.2018, Seite 30

5 Schlup J. Die Zukunft selber gestalten! Schweiz Ärztezeitung. 2018;99(1-2):3 